\title{
ARTICLE OPEN \\ A mechanism of defect-enhanced phase transformation kinetics in lithium iron phosphate olivine
}

\author{
Liang Hong ${ }^{1,2}$, Kaiqi Yang ${ }^{1,2}$ and Ming Tang (iD)
}

Antisite defects are a type of point defect ubiquitously present in intercalation compounds for energy storage applications. While they are often considered a deleterious feature, here we elucidate a mechanism of antisite defects enhancing lithium intercalation kinetics in $\mathrm{LiFePO}_{4}$ by accelerating the $\mathrm{FePO}_{4} \rightarrow \mathrm{LiFePO}_{4}$ phase transformation. Although $\mathrm{Fe}_{\mathrm{Li}}$ antisites block Li movement along the [010] migration channels in $\mathrm{LiFePO}_{4}$, phase-field modeling reveals that their ability to enhance Li diffusion in other directions significantly increases the active surface area for Li intercalation in the surface-reaction-limited kinetic regime, which results in order-of-magnitude improvement in the phase transformation rate compared to defect-free particles. Antisite defects also promote a more uniform reaction flux on (010) surface and prevent the formation of current hotspots under galvanostatic (dis)charging conditions. We analyze the scaling relation between the phase boundary speed, Li diffusivity and particle dimensions and derive the criteria for the co-optimization of defect content and particle geometry. A surprising prediction is that (100)-oriented LiFePO 4 plates could potentially deliver better performance than (010)-oriented plates when the Li intercalation process is surface-reactionlimited. Our work suggests tailoring antisite defects as a general strategy to improve the rate performance of phase-changing battery compounds with strong diffusion anisotropy.

npj Computational Materials (2019)5:118; https://doi.org/10.1038/s41524-019-0255-3

\section{INTRODUCTION}

Point, line and/or planar defects are ubiquitously present in all materials and frequently have beneficial effects on material properties. The intentional introduction and control of defects plays a key role in the development of advanced materials with better performance and new functionality. Well-known examples include doping semiconductors to modify the band structure and using phase or grain boundaries to strengthen alloys. Like other materials, battery intercalation compounds contain various types of defects. "Defect engineering" is a promising strategy for this class of materials, which nonetheless has not yet been widely explored. In particular, recent studies find that antisite defects, which are common in battery compounds, can promote $\mathrm{Li}$ transport and enhance rate performance by opening up alternative diffusion channels with lowered migration energies in numerous lithium-ion battery electrode materials. Such phenomena have been reported in $\mathrm{Li}_{1.211} \mathrm{Mo}_{0.467} \mathrm{Cr}_{0.3} \mathrm{O}_{2}{ }^{1}{ }^{1} \mathrm{Li}_{2}(\mathrm{Mn}$, Fe) $\mathrm{P}_{2} \mathrm{O}_{7}{ }^{2}$ a-LiMn ${ }_{1-x} \mathrm{Fe}_{x} \mathrm{PO}_{4}$ (ref. ${ }^{3}$ ) and $\mathrm{Li}_{4} \mathrm{Ti}_{5} \mathrm{O}_{12}$, etc. In refs. ${ }^{5,6}$, antisite defects are also reported to improve the stability and cyclability of cubic $\mathrm{Li}_{x} \mathrm{Ti}_{2} \mathrm{O}_{4}$, where the random mixing of $\mathrm{Li}$ and $\mathrm{Ti}$ on octahedral sites in the cubic phase electrochemically induced from amorphous $\mathrm{TiO}_{2}$ enables reversible capacity that cannot be achieved otherwise. These examples demonstrate that the rational tailoring of antisite defects provides a potentially general approach to improving battery electrode properties.

Here we present a computational study that reveals a new mechanism of antisite defects enhancing the rate capability of intercalation compounds by accelerating surface-reaction-limited (SRL) phase transformation during battery charge/discharge. Antisite defects are generated when the sites of intercalating ions are occupied by other cations that are usually less mobile. A prominent effect of antisite defects in battery electrodes is to block the existing paths of intercalating ions and generate new migration pathways at the same time. For intercalation compounds with strong anisotropic transport properties, which are common among battery materials, such effect usually leads to the reduction in the diffusion anisotropy of intercalating ions. For example, lithium iron phosphate olivine $\left(\mathrm{LiFePO}_{4}\right)$ is theoretically predicted to have predominantly one-dimensional (1D) Li diffusion in the [010] direction. ${ }^{7}$ However, experiments show that practically synthesized $\mathrm{LiFePO}_{4}$ with just a few percent of $\mathrm{Li}-\mathrm{Fe}$ antisite defects (Li/Fe) instead exhibit two-dimensional (2D) $\mathrm{Li}$ diffusivity. ${ }^{8,9}$ This discrepancy is explained by first-principles calculation $^{10}$ that finds $\mathrm{Li} / \mathrm{Fe}$ to impede $\mathrm{Li}$ movement along [010] migration channels but facilitate Li hopping between the channels via vacancies on Fe sites created by antisite defects. Similar observations of antisite defects reducing ion diffusion anisotropy are also reported for $\mathrm{Li}_{2} \mathrm{MP}_{2} \mathrm{O}_{7}$ (ref. $\left.{ }^{2}\right), \mathrm{Li}_{4} \mathrm{Ti}_{5} \mathrm{O}_{12}$ (ref. ${ }^{4}$ ), $\mathrm{Na}_{2+\delta} \mathrm{Fe}_{2-\delta / 2}\left(\mathrm{SO}_{4}\right)_{3}{ }^{11}$ and $\mathrm{LiMnBO}_{3} .{ }^{12}$

In this work, we show that antisite defects in $\mathrm{LiFePO}_{4}$ lead to an unexpected increase in the SRL phase transition rate. This is achieved by an increase in the surface reaction area for $\mathrm{Li}$ intercalation due to defect-enhanced Li diffusion along [100] even though antisites impede Li movement in the fast [010] diffusion direction. Analysis of the interplay between surface reaction and $\mathrm{Li}$ diffusion reveals that the inclusion of antisite defects in $\mathrm{LiFePO}_{4}$ qualitatively changes the particle size dependence of phase transformation rate in the $\mathrm{SRL}$ regime. As a result, the rate performance of defect-containing $\mathrm{LiFePO}_{4}$ is less sensitive to particle dimensions, which facilitates the use of larger particles to improve the packing density and reduce side reaction of the electrodes. Under galvanostatic conditions, the presence of antisites also reduces the risk of electrode damage from current hotspots by distributing the reaction flux more uniformly on particle surface. Due to the kinetic competition between surface reaction and bulk diffusion, an optimal defect concentration is 


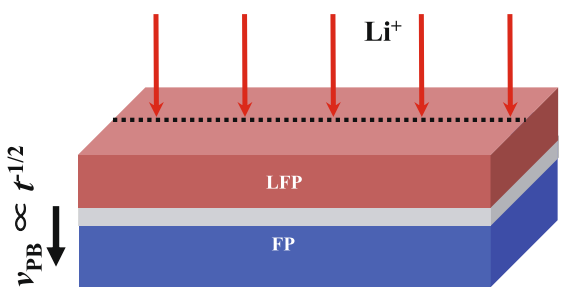

Bulk Diffusion Limited (BDL)

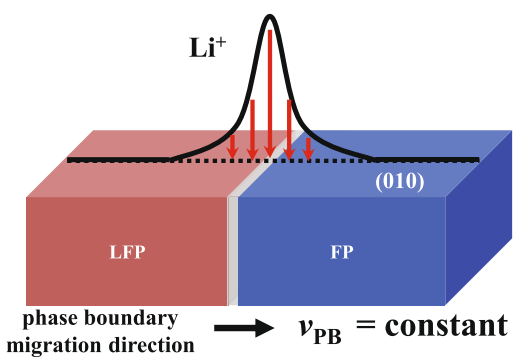

Surface Reaction Limited (SRL)

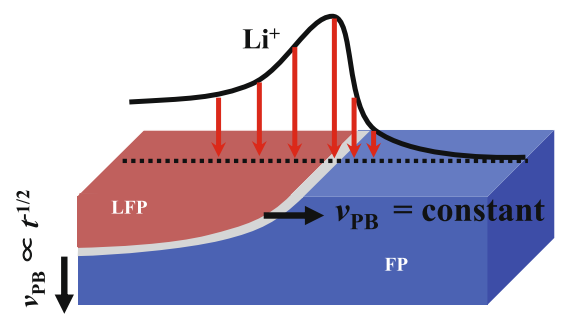

Hybrid

Fig. 1 Schematics of three distinct kinetic modes of phase transformation in $\mathrm{LiFePO}_{4}$.

predicted to exist for a given particle geometry to maximize the $\mathrm{Li}$ (de)intercalation rate. Criteria for the co-design of defect content and particle morphology are proposed. Counterintuitively, we find that (100)-oriented $\mathrm{LiFePO}_{4}$ platelike particles may exhibit even better rate performance than (010)-oriented plate particles in the $\mathrm{SRL}$ kinetic regime at relatively low defect levels, whereas the latter is commonly viewed as the most desirable $\mathrm{LiFePO}_{4}$ particle morphology. While we demonstrate the possibility of using antisite defects to accelerate phase transitions in $\mathrm{LiFePO}_{4}$, it may have general applicability to other phase-changing battery materials that exhibit ion diffusion anisotropy.

\section{RESULTS}

Electrochemically driven first-order phase transformations in battery electrode materials upon ion (de)intercalation are subject to the kinetic control of various rate-limiting steps. We previously show $^{9}$ that the competition between Li diffusion and surface reaction can give rise to three distinct phase transformation modes in $\mathrm{LiFePO}_{4}$, i.e. bulk-diffusion-limited (BDL), SRL and an intermediate hybrid mode, in which phase boundary migration is $\mathrm{BDL}$ or SRL in different directions. As illustrated in Fig. 1, these transformation modes are associated with different phase growth morphologies and rates. They are not unique to $\mathrm{LiFePO}_{4}$ and can operate in other intercalation compounds. Phase transformation becomes SRL when Li insertion/extraction at the electrode particle surface is much more sluggish than its diffusion inside the particles. This could occur at small particle sizes, low applied over/ under-potentials and/or low exchange current density for charge transfer, etc. ${ }^{9}$ In a pioneering study, Singh, Ceder and Bazant (SCB) investigated the SRL phase transformation kinetics using a depthaveraged model of $\mathrm{LiFePO}_{4}{ }^{13}$ They show that a salient feature of the SRL mode is that phase boundary travels at a constant velocity perpendicular to the main Li intercalation direction (Fig. 1), which is in sharp contrast to the behavior of the BDL mode. Although the SCB theory assumes 1D Li transport in $\mathrm{LiFePO}_{4}$ in accordance with the DFT prediction, ${ }^{7}$ the characteristics of the SRL mode are independent of Li diffusion anisotropy and also apply to cases with higher dimensional Li diffusivity in the presence of antisite defects. ${ }^{9}$

Antisite defects accelerate SRL phase transformation

We study the SRL phase transformation process in defectcontaining $\mathrm{LiFePO}_{4}$ particles using the mesoscale phase-field simulation method, ${ }^{14-16}$ which has been applied to the modeling of LiFePO $4 .{ }^{17-21}$ An unexpected phenomenon that antisite defects can increase the phase boundary migration speed is observed from the simulations, in which the influence antisite defects exert on the discharge process is captured by their effect on the anisotropic Li diffusivity in $\mathrm{LiFePO}_{4}$. In ref. ${ }^{10}$, Malik et al. evaluate from first-principles calculation the activation barrier required for $\mathrm{Li}^{+}$to cross over to a neighbor channel through an antisite in $\mathrm{LiFePO}_{4}$. The calculated $\mathrm{Li}$ migration barrier is employed to

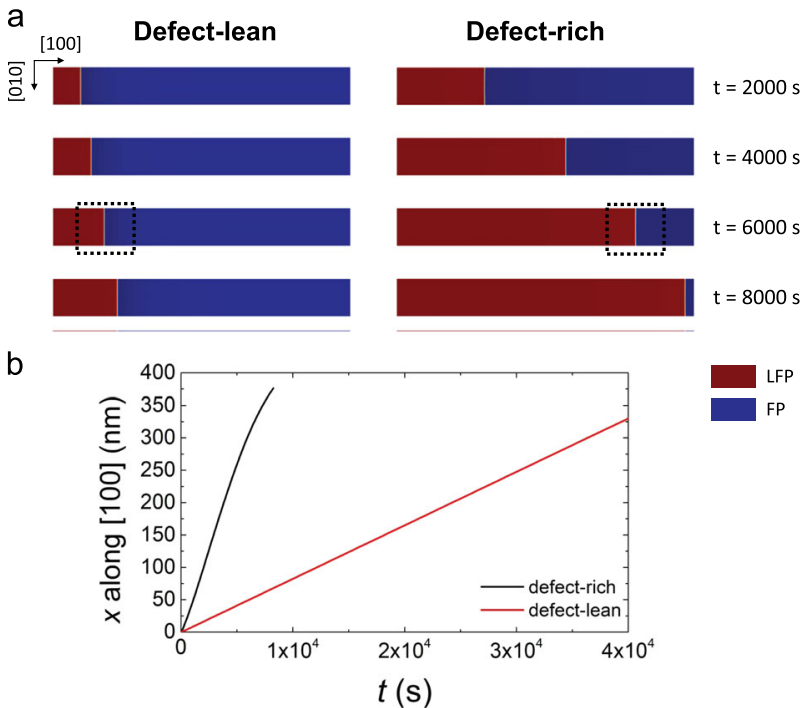

Fig. 2 Phase-field simulations of SRL phase boundary movement in defect-rich ( $25 \%$ antisites) vs defect-lean $(0.5 \%$ antisites) $(010)$ oriented $\mathrm{LiFePO}_{4}$ particles of size $L_{[100]} \times L_{[010]}=400 \mathrm{~nm} \times 50 \mathrm{~nm}$ under underpotential $\Delta \phi=35 \mathrm{mV}$. $\mathrm{Li}$ is assumed to be inserted into the particles from (010) surfaces only and no-flux boundary condition is applied to other surfaces. a Snapshots at time $=2000$, 4000,6000 and $8000 \mathrm{~s}$. The dashed black boxes indicate the regions showing bulk diffusion flux distributions in Fig. 3b. b Phase boundary location along [100] vs time.

determine the defect concentration dependence of Li diffusivity along [010], [100] and [001] axes, $D_{[010]}, D_{[100]}$ and $D_{[001]}$, at $440 \mathrm{~K}$. While $D_{[010]}$ at the room temperature $(300 \mathrm{~K})$ is also reported at several defect levels in ref. ${ }^{10}$, the values of $D_{[100]}$ and $D_{[001]}$ at $300 \mathrm{~K}$ are not provided in that work. As they are required for our simulations, we calculated all the three diffusion coefficients at $300 \mathrm{~K}$ as a function of antisite concentration following the approach of Malik et al. ${ }^{10}$ (see Methods). As shown in Supplementary Fig. 1 in the Supplementary Information (SI), $D_{[100]}$ exhibits an approximately inverse relationship with $D_{[010]}$ at $300 \mathrm{~K}$. Both of them approach $10^{-12}-10^{-11} \mathrm{~cm}^{2} \mathrm{~s}^{-1}$ at high defect concentrations, consistent with our previous observation of $2 \mathrm{D}$ room-temperature Li diffusivity $D_{[010]} \approx D_{[100] /[001]} \approx 10^{-11} \mathrm{~cm}^{2} \mathrm{~s}^{-1}$ in defect-containing $\mathrm{LiFePO}_{4}$ microparticles. ${ }^{9}$ The calculated $\mathrm{Li}$ diffusivity values at $300 \mathrm{~K}$ are used in the phase-field simulations presented below.

Figure 2 compares the discharge simulations for two $\mathrm{LiFePO}_{4}$ particles with different antisite contents at a constant underpotential $\Delta \phi=35 \mathrm{mV}$, which is below the underpotential required to form a metastable solid solution in $\mathrm{LiFePO}_{4}$. The particles are given (010)-oriented platelike shape in simulations to facilitate fast Li diffusion along the [010] direction and ensure that the phase transition is in the SRL regime. Such particle morphology is 


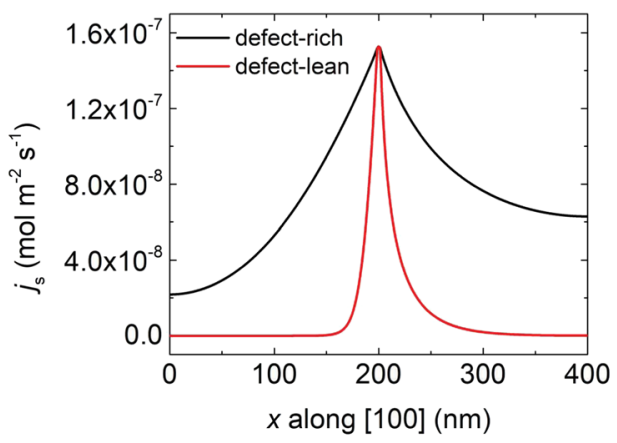

b

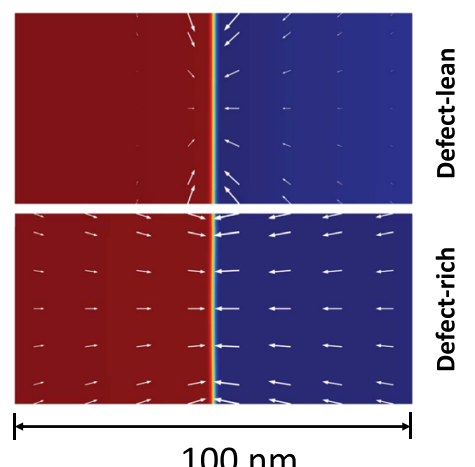

Fig. 3 Comparison of Li flux distributions in defect-lean vs defect-rich particles. a Li intercalation flux $j_{s}$ on $(010)$ particle surface when phase boundary is at the particle center. $\mathbf{b}$ Li bulk diffusion flux distribution inside a region within $50 \mathrm{~nm}$ from the phase boundary. Arrow length is proportional to the magnitude of flux. To make the flux distribution in the defect-lean particle more visible, its scaling factor is set five times larger than in defect-rich particle.

commonly obtained in hydrothermal synthesis. ${ }^{22,23}$ In the simulations, $\mathrm{Li}$ is inserted into the particle from (010) surface only, and zero-flux boundary condition is applied to (100) surfaces. The "defect-lean" particle contains $0.5 \%$ antisites and has a large $D_{[010]}$ $=1.3 \times 10^{-10} \mathrm{~cm}^{2} \mathrm{~s}^{-1}$ but a much smaller $D_{[100]}=3.9 \times 10^{-14} \mathrm{~cm}^{2}$ $\mathrm{s}^{-1}$. The "defect-rich" particle contains $25 \%$ antisites and has a much reduced $\mathrm{Li}$ diffusion anisotropy with $D_{[010]}=5.4 \times 10^{-12}$ $\mathrm{cm}^{2} \mathrm{~s}^{-1}$ and $D_{[100]}=2.6 \times 10^{-12} \mathrm{~cm}^{2} \mathrm{~s}^{-1}$. Because phase boundaries are observed to be parallel to the [001] axis in platelike particles, ${ }^{18,22,24,25}$ simulations were reduced to two dimensions to improve computation efficiency. Coherency stress that arises from the lattice mismatch between $\mathrm{LiFePO}_{4}$ (LFP) and $\mathrm{FePO}_{4}$ (FP) phases plays a critical role in inducing the metastable solid solution behavior at high (dis)charge rates. ${ }^{18,26}$ However, it is found that the omission of coherency stress does not cause any significant change on the phase transformation kinetics in the SRL regime (see Supplementary Fig. 2), and stress is thus not considered in simulations to simplify the theoretical analysis described later. As shown in Fig. 2, the FP $\rightarrow$ LFP phase transition in both particles is in the SRL mode, with the phase boundary traveling along [100] at a constant speed. However, the phase boundary velocity in the defect-rich particle is six times higher than in the defect-lean particle, even though the Li diffusivity in the main intercalation direction [010] is two decades larger in the latter. We also performed similar simulation for SRL phase transformation at $\mathrm{T}=440 \mathrm{~K}$ using the defect-concentrationdependent Li diffusivity values at this temperature reported in ref. ${ }^{10}$. Supplementary Fig. 3 shows a similar increase of the phase boundary speed by one order of magnitude in $\mathrm{LiFePO}_{4}$ containing $10 \%$ defects at $440 \mathrm{~K}$. This finding is surprising as SRL phase transformation is expected to be kinetically limited by surface reaction and insensitive to Li diffusion kinetics.

A revealing clue to this counter-intuitive result can be found in Fig. 3a, which shows the distribution of Li intercalation flux $j_{s}$ on (010) surface in both particles. Li insertion in the defect-lean particle only takes place near the phase boundary and $j_{s}$ decays rapidly away from the boundary center. The narrow surface reaction region moves together with the phase boundary, resulting in the sequential filling of [010] Li channels along the [100] direction during discharge. Such behavior is analogous to the prediction of SCB theory and the "domino-cascade" model proposed by Delmas et al. ${ }^{27}$ In contrast, the entire (010) surface actively intercalates $\mathrm{Li}$ in the defect-rich particle. While its peak value is the same as that in the defect-lean particle, $j_{s}$ decreases more slowly away from the phase boundary. As such, more Li ions are inserted into the defect-rich particle per unit time, which explains its higher phase transformation rate. Figure $3 \mathrm{~b}$ shows that the wider surface reaction region on the defect-rich particle is a direct result of the enhanced [100] diffusivity enabled by defects. It can be seen that the Li diffusion flux in the defect-lean particle is confined near phase boundary. However, significant Li diffusion flux along [100] exists within the entire defect-rich particle, which transports $\mathrm{Li}$ atoms inserted at surface locations distant from the phase boundary to the boundary to participate in phase transformation. This results in a larger surface reaction area. Therefore, a key insight we obtained is that an increased $\mathrm{Li}$ diffusivity along the phase boundary migration direction ([100] here) benefits the SRL phase transformation kinetics in $\mathrm{LiFePO}_{4}$ by expanding the surface reaction region. This differs from the effect of faster Li diffusion on improving phase transformation kinetics in the $\mathrm{BDL}$ regime, in which a higher diffusivity enhances phase boundary velocity in the same direction. It represents a new mechanism of accelerating phase transitions through the interplay between Li diffusion and surface reaction, which has not been explored so far.

To further shed light on the effect of Li diffusion on SRL kinetics, especially the quantitative dependence of phase boundary velocity on [100] and [010] Li diffusivity, a series of particle discharge simulations under constant $\Delta \phi=35 \mathrm{mV}$ were carried out. Instead of obeying the relation given by Supplementary Fig. 1, the values of $D_{[010]}$ and $D_{[100]}$ are independently varied in simulations in order to study their respective effects on the phase transition kinetics. The [010] thickness of the particle is $L_{[010]}=$ $50 \mathrm{~nm}$, and its [100] dimension $L_{[100]}$ is assumed to be much larger than the surface reaction region so that the phase boundary velocity $V_{\mathrm{PB}}$ is independent of $L_{[100]}$. As shown in Fig. 4, varying $D_{\text {[010] }}$ from $10^{-9}$ to $10^{-12} \mathrm{~cm}^{2} \mathrm{~s}^{-1}$ at a given $D_{[100]}$ has little effect on the calculated phase boundary velocity $V_{\mathrm{PB}}$ and the surface reaction zone width $W$, which is defined as the width of the region where $j_{\mathrm{s}}$ is larger than $35 \%$ of its peak value. This confirms that phase transition is indeed in the SRL mode and hence insensitive to $D_{[010]}$. We note that $D_{[010]}$ in defect-containing $\mathrm{LiFePO}_{4}$ is predicted to increase beyond the bulk diffusivity value at very small [010] particle thickness when antisites can no longer block the 1D migration channels. ${ }^{10}$ The results above suggest that this particle size dependence of $D_{[010]}$ does not have a large impact on the predicted acceleration of phase transition by antisites in the SRL regime. On the other hand, increasing $D_{[100]}$ from $10^{-14}$ to $10^{-11} \mathrm{~cm}^{2} \mathrm{~s}^{-1}$ while keeping $D_{[010]}$ constant causes both $W$ and $V_{\mathrm{PB}}$ to increase by more than 20 times.

\section{Depth-averaged model}

We found that the $2 \mathrm{D}$ simulation results can be well approximated by a $1 D$ depth-averaged model similar to the SCB theory, which is derived by assuming that $\mathrm{Li}$ concentration $\mathrm{C}$ is uniform along [010] 


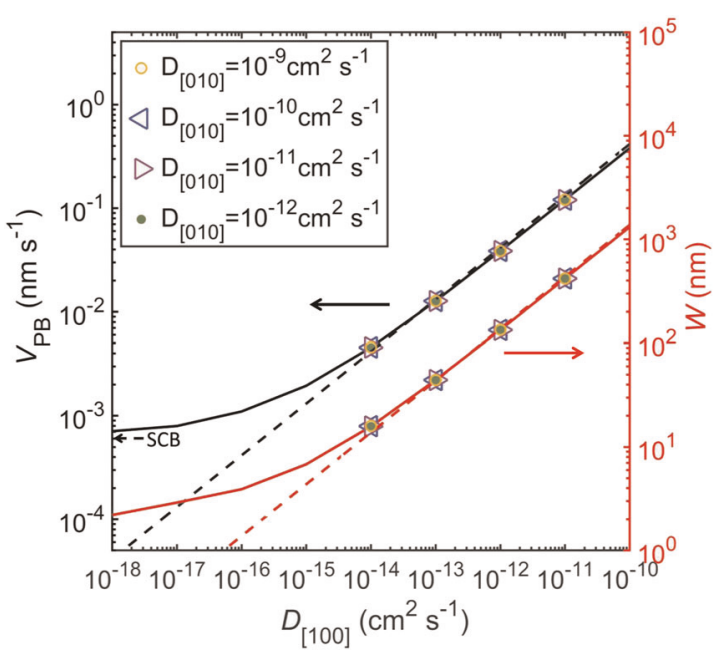

Fig. 4 Dependence of phase boundary velocity $V_{\mathrm{PB}}$ and surface reaction zone width $W$ on $D_{[010]}$ and $D_{[100]}$. Markers represent $2 D$ phase-field simulation results. Solid and dashed lines are numerical and approximate analytical solutions to the depth-averaged model, respectively. Dashed arrow indicates the $V_{\mathrm{PB}}$ value predicted by the SCB model, i.e. for the case of $D_{[100]}=0$.

and a function of [100]-coordinate $x$ only. With the simplification, the governing equation of the phase-field model becomes

$$
\frac{\partial c}{\partial t}=\frac{\partial}{\partial x}\left[\frac{D_{[100]} V_{\mathrm{m}}}{R T} c(1-c) \frac{\partial \mu_{\mathrm{Li}}}{\partial x}\right]+\frac{2 j_{\mathrm{s}}}{L_{[010]}}
$$

where the surface reaction flux $j_{s}$ on one (010) facet is given by the Butler-Volmer equation (Methods). Equation 1 has the form of a reaction-diffusion equation. It reduces to the SCB model when the diffusion term disappears from Eq. 1 at $D_{[100]}=0$. Phase boundary velocity $V_{\mathrm{PB}}$ and surface reaction zone width $W$ predicted by this model are shown as solid lines in Fig. 4. The excellent agreement with the full simulations allows one to use the depth-averaged model to efficiently analyze the SRL kinetics in the presence of [100] Li diffusion.

\section{Scaling relation}

The simulation results in Fig. 4 show that both $V_{\mathrm{PB}}$ and $W$ hold the same parabolic relation with $D_{[100]}$, i.e. $V_{\mathrm{PB}} \propto D_{[100]}^{1 / 2}$ and $W \propto D_{[100]}^{1 / 2}$, when $D_{[100]}>\sim 10^{-14} \mathrm{~cm}^{2} \mathrm{~s}^{-1}$. Furthermore, $V_{\mathrm{PB}}$ is proportional to $W$ over the entire range of $D_{[100]}$. When $D_{[100]} \rightarrow 0, W$ approaches the intrinsic diffuse interface width in the phase-field model, and $V_{\mathrm{PB}}$ reaches the prediction of the SCB model. It shows that a defect-free LFP particle has a very low SRL phase boundary velocity $\left(<10^{-3} \mathrm{~nm} \mathrm{~s}^{-1}\right)$ because of a very narrow surface reaction zone $(\sim 2 \mathrm{~nm})$. As a result, good rate performance can only be achieved when the FP $\rightarrow$ LFP first-order phase transition is bypassed. However, when an adequate amount of antisite defects are present in the particle, the surface reaction area can increase by orders of magnitude and results in a similar increase in the phase boundary velocity to enable much better rate capability in the absence of metastable solid solution.

A more transparent understanding of the scaling relation can be obtained from an approximate analytical solution to the depthaveraged model, which is based on the sharp-interface assumption (see derivation in Methods). The solution gives the following expressions of surface reaction zone width $W$ and phase boundary

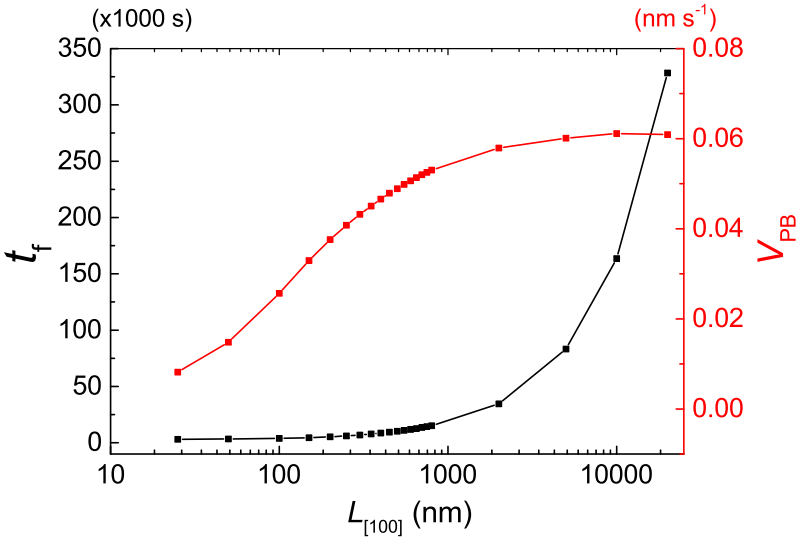

Fig. 5 Effect of $L_{[100]}$ on the average phase boundary velocity $V_{\mathrm{PB}}$ (red line) and total transformation time $t_{f}$ (black line) for Li intercalation into a particle with $D_{[100]}=2.6 \times 10^{-12} \mathrm{~cm}^{2} \mathrm{~s}^{-1}$ at $\Delta \phi=35 \mathrm{mV}$. The [010] particle size is $50 \mathrm{~nm}$.

velocity $V_{\mathrm{PB}}$ :

$W=\lambda_{1} \sqrt{\frac{D_{[100]} L_{[010]}}{i_{0}}}$

$V_{\mathrm{PB}}=\lambda_{2} \sqrt{\frac{i_{0} D_{[100]}}{L_{[010]}}}$

where the expressions of $\lambda_{1}$ and $\lambda_{2}$, which are functions of $\Delta \phi$, are given in Methods, and $i_{0}$ is the exchange current density. As shown in Fig. 4, Eqs. 2 and 3 show excellent agreement with the numerical solution when $W$ is significantly larger than the diffuseinterface width of the phase boundary. In addition to explaining the parabolic dependence of $W$ and $V_{\mathrm{PB}}$ on $D_{[100]}$, Eq. 3 shows that $V_{\mathrm{PB}}$ varies with $i_{0}$ and [010] particle thickness $L_{[010]}$ as $i_{0}{ }^{1 / 2}$ and $L_{[010]}^{-1 / 2}$, respectively. Notably, these relations qualitatively differ from defect-free LFP particles with 1D Li diffusivity, in which $V_{\mathrm{PB}}$ has a stronger dependence on $i_{0}$ and $L_{[010]}$, i.e. $V_{\mathrm{PB}} \propto i_{0}$ and $V_{\mathrm{PB}} \propto L_{[010]}^{-1}$, according to the SCB theory. Such difference in the scaling behavior lies in that upon decreasing $i_{0}$ or increasing $L_{[010]}$, the surface reaction area $W$ increases in defect-containing particles (Eq. 2), which contributes to a less pronounced reduction of $V_{\mathrm{PB}}$.

Particle size dependence of phase transformation time

The phase boundary speed predicted by Eq. 3 applies to the situation where the [100] particle size is larger than the surface reaction zone width $W$. When $L_{[100]}<W$, the entire (010) particle surface is active for $\mathrm{Li}$ intercalation during discharge. An interesting prediction thus arises: $V_{\mathrm{PB}}$ should increase approximately linearly with $L_{[100]}$ in the $S R L$ regime, i.e. the longer the travel distance of the phase boundary, the faster it moves. Accordingly, the particle transformation time, given by $t_{\mathrm{f}} \approx L_{[100]} / V_{\mathrm{PB}}$, should be insensitive to $L_{[100]}$. This prediction is confirmed by calculations shown in Fig. 5, which plots the average $V_{\mathrm{PB}}$ and $t_{\mathrm{f}}$ as a function of $L_{[100]}$ for $L i$ intercalation into a defect-rich (25\% antisites) particle at $\Delta \phi=35 \mathrm{mV}$. It can be seen that $V_{\mathrm{PB}}$ is proportional to $L_{[100]}$ and $t_{\mathrm{f}}$ increases very slowly with $L_{[100]}$ up to $\sim 1 \mu \mathrm{m}$, which makes $t_{\mathrm{f}}$ only dependent on [010] particle size as $t_{\mathrm{f}} \propto \sqrt{L_{[010]}}$. In contrast, $t_{\mathrm{f}}$ is proportional to $L_{[100]} L_{[010]}$ in defect-free particles. Along with Eq. 3, this comparison shows that the inclusion of antisite defects results in qualitatively different particle size dependence of the SRL phase transition kinetics. The weaker dependence of $t_{f}$ on both $L_{[100]}$ and $L_{[010]}$ in defect-containing particles implies another benefit of antisite defects, i.e. they enable the rate performance of $\mathrm{LiFePO}_{4}$ to degrade less severely with particle dimensions. This facilitates the use of larger particles in 
applications, which can improve the packing density and reduce side reactions between particles and electrolyte in electrodes.

Galvanostatic cycling behavior

Besides constant underpotential condition, we also studied the difference between defect-rich and defect-lean particles under galvanostatic discharge or constant current conditions. Figure $6 a$ compares the distributions of $\mathrm{Li}$ intercalation flux $j_{\mathrm{s}}$ on the (010) surface of particles with different defect contents when
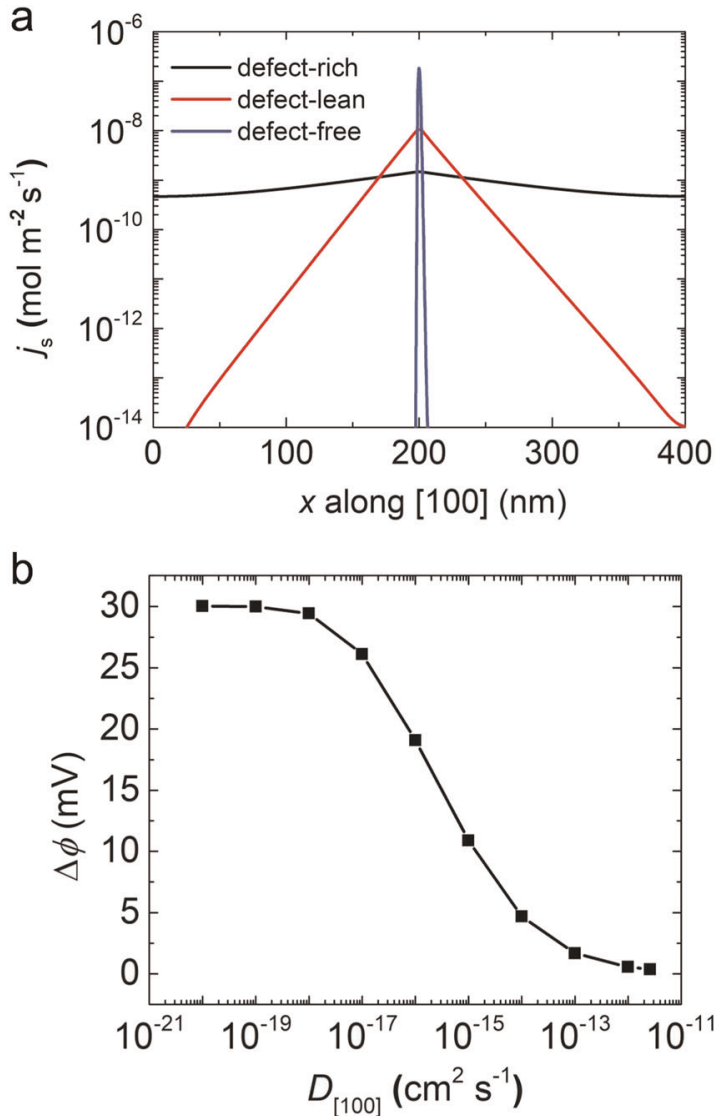

Fig. 6 Effect of $D_{[100]}$ on SRL phase transformation behavior in a particle of size $L_{[100]} \times L_{[010]}=400 \mathrm{~nm} \times 50 \mathrm{~nm}$ galvanostatically discharged at $0.005 \mathrm{C}$. a $\mathrm{Li}$ intercalation flux distribution $j_{s}$ in logarithmic scale at $50 \%$ state of charge in defect-rich, defect-lean and defect-free particles, in which $D_{[100]}=2.6 \times 10^{-12}, 3.9 \times 10^{-14}$ and $0 \mathrm{~cm}^{2} \mathrm{~s}^{-1}$, respectively. b Dependence of the underpotential applied to the particle on $D_{[100]}$. galvanostatically discharged to $50 \%$ state of charge at $0.005 \mathrm{C}$ ( $n \mathrm{C}=$ fully discharged in $1 / n$ hours). While the total amount of $\mathrm{Li}$ intercalated into the particles per unit time is the same, the peak value of $j_{s}$ decreases sharply with $D_{[100]}$ because faster [100] diffusion causes the reaction flux to be more evenly distributed on (010) surface. A 123-fold reduction in the peak flux occurs when $D_{\text {[100] }}$ increases from $0 \mathrm{~cm}^{2} \mathrm{~s}^{-1}$ ( $0 \%$ antisites) to $2.6 \times 10^{-12} \mathrm{~cm}^{2} \mathrm{~s}^{-1}$ (25\% antisites). Meanwhile, increasing $D_{[100]}$ also significantly decreases the underpotential required from $30 \mathrm{mV}$ to $0.3 \mathrm{mV}$ as shown in Fig. 6b. Therefore, antisite defects are beneficial under galvanostatic cycling conditions by reducing polarization and mitigating degradation caused by current hotspots and electrochemical shock. ${ }^{28-30}$

Optimization of defect concentration and particle morphology After demonstrating the benefits of antisite defects on phase transformation kinetics in the SRL regime, we ask the question whether there exists an optimal defect concentration and how it depends on the $\mathrm{LiFePO}_{4}$ particle geometry. We still consider the platelike particle morphology here for its practical relevance. The SRL transformation rate is maximized when the entire plate surface is active for Li intercalation during (dis)charge, which requires $L_{[100]}<W$. Using Eq. 2, this leads a criterion on [100] Li diffusivity

$D_{[100]}>\frac{i_{0} L_{[100]}^{2}}{\lambda_{1} L_{[010]}}$

On the other hand, Li bulk diffusion along the [010] plate thickness direction should be sufficiently facile so that it does not limit the transformation kinetics. This condition can be described by the inequality $L_{[010]}^{2} / D_{[010]}<W / V_{\mathrm{PB}}$, where $L_{[010]}^{2} / D_{[010]}$ is the characteristic Li diffusion time along the [010] axis and $W / V_{\mathrm{PB}}$ is the time a [010] channel stays active for Li intercalation. Applying Eqs. 2 and 3, we derive a criterion on [010] Li diffusivity

$D_{[010]}>\frac{\lambda_{2} i_{0} L_{[010]}}{\lambda_{1}}$

Equations 4 and 5 provide the guidance on tuning the defect concentration for given particle sizes, or conversely, the particle geometry for a given defect content.

Because increasing antisite defect concentration has opposite effects on $D_{[100]}$ and $D_{[010]}$, Eqs. 4 and 5 may not always be satisfied simultaneously and an optimal defect level may exist. We numerically examine the defect concentration dependence of SRL transformation rate in 2D phase-field simulations, in which a (010)oriented $\mathrm{LiFePO}_{4}$ particle of $L_{[100]} \times L_{[010]}=400 \mathrm{~nm} \times 50 \mathrm{~nm}$ is discharged at $\Delta \phi=35 \mathrm{mV}$. Figure 7 shows that the lithium intercalation time keeps decreasing with antisite concentration up

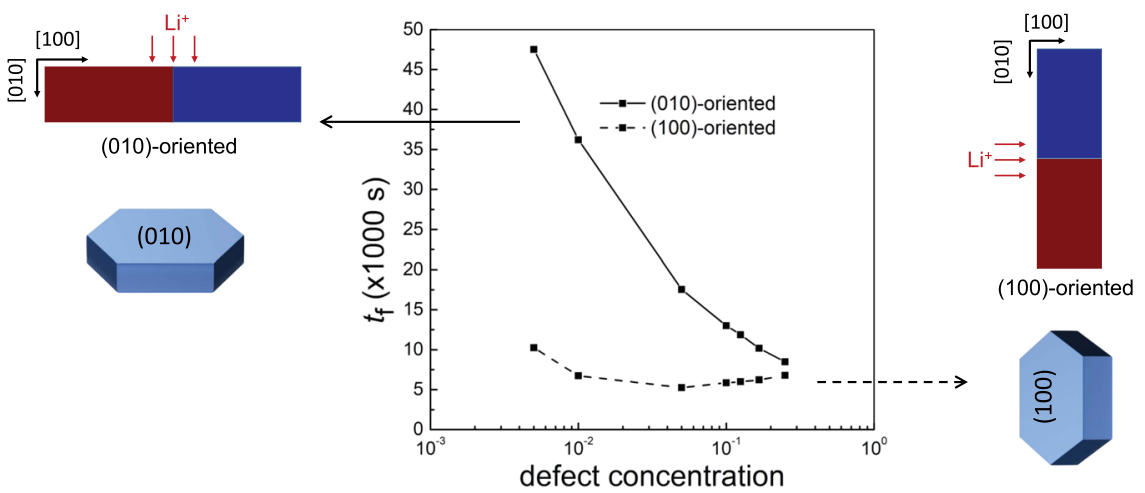

Fig. 7 Optimization of defect concentration and particle morphology for SRL phase transformation kinetics. The total transformation time $t_{\mathrm{f}}$ is plotted as a function of $D_{[100]}$ or $D_{[010]}$ for (010)-oriented $\left(L_{[100]} \times L_{[010]}=400 \times 50 \mathrm{~nm}\right.$, solid line) and (100)-oriented $\left(L_{[100]} \times L_{[010]}=\right.$ $50 \times 400 \mathrm{~nm}$, dashed line) platelet particles discharged at $\Delta \phi=35 \mathrm{mV}$. 
to $25 \%$, which implies an optimal defect level at higher defect levels that nonetheless may be practically unfeasible to realize. However, we find that comparable performance may be attained at much lower defect concentrations by changing the particle shape from (010)-oriented to (100)-oriented plates. For such particle morphology, the main reaction surface is the (100) facet, which is observed to be active for Li intercalation in defectcontaining LFP particles in recent in situ TXM experiments., ${ }^{9,31}$ Because SRL phase boundary movement is parallel to the [010] fast Li diffusion direction in (100)-oriented plates, the phase boundary velocity could be significantly improved over (010)oriented plates at the same defect concentration. This is confirmed by simulations shown in Fig. 7. For a (100)-oriented particle with $L_{[100]} \times L_{[010]}=50 \mathrm{~nm} \times 400 \mathrm{~nm}$, the minimum intercalation time is reached at $5 \%$ defect concentration. We note that this result is obtained assuming that the exchange current density $i_{0}$ for $\mathrm{Li}$ intercalation is the same for (100) and (010) surfaces, which is taken to be $0.01 \mathrm{~A} \mathrm{~m}^{-2}$ based on estimate from ref. ${ }^{32}$ While direct measurement of $i_{0}$ on the (100) facet is not available in literature, experimental observations of Li intercalation on non(010) surfaces in $\mathrm{LiFePO}_{4}{ }^{9,31}$ implies that it is comparable to that of (010) surface. For example, the hybrid-mode phase boundary migration speed on $(100) /(001)$ surfaces in a $\mathrm{LiFePO}_{4}$ microrod sample is fitted with $i_{0}=0.1 \mathrm{~A} \mathrm{~m}^{-2}$ in ref. ${ }^{9}$. Therefore, without introducing excessive amount of defects, (100)-oriented plates could potentially provide better performance in the SRL regime than (010)-oriented plates, which are usually assumed to be the desired particle shape for fast (dis)charging. Interestingly, a recent study indeed finds (100)-oriented LFP nanoplates to exhibit excellent rate capability $^{33}$ even better than (010)-oriented nanoparticles, ${ }^{34}$ which may be related to the defect-based mechanism discussed here.

As a commercial cathode material for $\mathrm{Li}$-ion batteries, $\mathrm{LiFePO}_{4}$ is known for its exceptional rate capability. Recent experiments $^{26,35,36}$ and modeling studies ${ }^{18,37,38}$ establish the formation of metastable solid solution in $\mathrm{LiFePO}_{4}$ during fast (dis)charge, and it is widely believed that bypassing the sluggish first-order phase transition is responsible for considerably accelerating the $\mathrm{Li}$ intercalation kinetics. Here we demonstrate a different, defectbased acceleration mechanism without the suppression of the FP $\rightarrow$ LFP phase transformation. Compared to the former, such mechanism could be effective at low overpotentials where the metastable solid solution does not form. This could be attractive for battery operation where the magnitude of applied overpotential is limited. For example, the development of thickelectrode battery cells has gained significant interest recently as a way to improve energy density. ${ }^{39-41}$ However, thick electrodes are often plagued by severe reaction non-uniformity as a wide range of overpotentials typically exists across the electrodes due to electrolyte polarization. ${ }^{41}$ The inability of LFP particles to form solid solution in the low overpotential region is likely to exacerbate the nonuniform reaction and cause capacity underutilization. Using defect-containing LFP particles in thick electrodes could alleviate this issue. The enhancing effect of defects on SRL phase transformation rate also offers a promising way to improve the performance of electrode materials that do not exhibit metastable solid solution behavior and/or have very sluggish surface reaction kinetics.

In a recent work, ${ }^{42} \mathrm{Li}$ et al. reveal that fast Li surface diffusion on $\mathrm{LiFePO}_{4}$ surface facilitated by adsorbed fluids promotes $\mathrm{Li}$ redistribution within the (010) plane inside the particles and significantly enhances the intra-particle phase separation kinetics. There, Li surface diffusion plays a similar role of antisite defects in increasing the effective Li diffusivity along non-[010] directions. A reaction-diffusion equation similar to Eq. 1 is employed in ref. ${ }^{42}$ to analyze the stability of $\mathrm{Li}_{x} \mathrm{FePO}_{4}$ solid solution in the presence of $\mathrm{Li}$ surface diffusion. Although $\mathrm{Li}$ et al. focuses on the effect of fast surface diffusion on phase separation kinetics, it is also expected to increase the surface reaction area and accelerate SRL phase transformation in the two-phase coexistence regime, where phase separation is fully developed. This phenomenon can be analyzed within the same theoretical framework of this work by considering the contribution of surface diffusion to $D_{[100]}$ in Eq. 1. Li diffusivity on (010) $\mathrm{LiFePO}_{4}$ surface in contact with electrolyte is conservatively estimated to be $\sim 10^{-12} \mathrm{~cm}^{2} \mathrm{~s}^{-1}$, which leads to an effective in-plane diffusivity of $\sim 10^{-14} \mathrm{~cm}^{2} \mathrm{~s}^{-1}$ in 150nm-thick, (010)oriented $\mathrm{LiFePO}_{4}$ plates. ${ }^{42}$ Compared to $D_{[100]} \approx 10^{-12} \mathrm{~cm}^{2} \mathrm{~s}^{-1}$ in defect-rich particles, this represents a relatively small contribution to [100] Li transport although its importance will certainly increase with decreasing particle size, which warrants further study. Conversely, like Li surface diffusion antisite defects can also promote phase separation at high underpotentials where a metastable solid solution can form $(\Delta \phi>45 \mathrm{mV}$ in our model). In a phase-field modeling study, ${ }^{43}$ Dargaville and Farrell show that increasing [100] Li diffusivity induces phase separation in $\mathrm{LiFePO}_{4}$ under high discharge currents, which otherwise favor the solidsolution intercalation behavior. Antisite defects may have a beneficial effect on SRL intercalation kinetics even in this regime. For instance, Supplementary Fig. 4a compares the lithiation rate of defect-rich ( $25 \%$ antisites) vs defect-lean ( $0.5 \%$ antisites) particles under $\Delta \phi=50 \mathrm{mV}$. It shows that the former is fully lithiated at $\sim 8000 \mathrm{~s}$, when the latter only reaches $\sim 60 \%$ lithiation. The reason for such difference lies in that defect-enhanced [100] Li diffusion causes phase separation to initiate in $\mathrm{Li}_{x} \mathrm{FePO}_{4}$ metastable solid solution at an earlier time of $\sim 5700 \mathrm{~s}$ (see Supplementary Fig. 4b), after which the Li chemical potential at the particle surface is reduced to the LFP/FP two-phase equilibrium level. As shown in Supplementary Fig. 4b, this in turn increases the surface reaction overpotential in the Butler-Volmer kinetics to generate a larger $\mathrm{Li}$ intercalation flux. A detailed analysis of the interplay between antisites, phase separation and Li intercalation kinetics will be presented elsewhere.

Finally, we discuss potential experimental strategy to examine the predicted beneficial effects of antisite defects in $\mathrm{LiFePO}_{4}$. We suggest that our predictions could be tested experimentally by annealing hydrothermally synthesized $\mathrm{LiFePO}_{4}$ platelike particles at different temperatures to vary the defect level. In general, increasing the annealing temperature reduces the amount of defects in electrode materials. ${ }^{2,44-46}$ For instance, this approach has been demonstrated by Chen and Graetz, ${ }_{14}^{44}$ who report that the $\mathrm{Fe}_{\mathrm{Li}}$ antisite concentration in $\mathrm{LiFePO}_{4}$ prepared by a hydrothermal method decreases from 8 to $0 \%$ when the postsynthesis annealing temperature is increased from $440{ }^{\circ} \mathrm{C}$ to $500{ }^{\circ} \mathrm{C}$. In ref. ${ }^{2}$, Kim et al. synthesized $\mathrm{Li}_{2} \mathrm{MnP}_{2} \mathrm{O}_{7}$ with $\sim 0 \%, 20 \%$ and $30 \% \mathrm{Li} / \mathrm{Mn}$ antisites by calcinating samples at $700^{\circ} \mathrm{C}, 650^{\circ} \mathrm{C}$ and $600{ }^{\circ} \mathrm{C}$, respectively, which are tested to confirm the effect of antisite defects on promoting Li diffusion in this material.

\section{DISCUSSION}

In summary, we reveal that the inclusion of antisite defects in $\mathrm{LiFePO}_{4}$ particles could result in orders-of-magnitude improvement in SRL phase transformation kinetics by increasing the active surface area for $\mathrm{Li}$ intercalation. Such phenomenon originates from the experimentally confirmed effect of antisite defects on reducing the $\mathrm{Li}$ diffusion anisotropy and is expected to be applicable to other phase-changing intercalation compounds with anisostropic ion transport properties. We numerically and analytically study the interplay between Li diffusion and surface reaction in the SRL regime. The results show that antisite defects qualitatively change the scaling dependence of the phase transformation rate on $\mathrm{Li}$ diffusivity, exchange current density and particle size. Upon potentiostatic discharge, the Li intercalation rate deteriorates more slowly with increasing particle dimensions in defect-rich particles, which facilitates the use of larger electrode particles without severely compromising rate 
performance. Antisites also induce a more uniform distribution of the reaction flux on particle surface under galvanostatic discharge conditions, which reduces the risk of electrode degradation due to electrochemical shock. We show that the optimal defect concentration that maximizes $\mathrm{Li}$ intercalation rate is dependent on particle geometry, and (100)-oriented platelike $\mathrm{LiFePO}_{4}$ potentially offers better rate performance than (010)-oriented plates during SRL (dis)charge process. Our work highlights the promise and opportunities of improving battery electrode compounds through intentional defect manipulation guided by a mechanistic understanding.

\section{METHODS}

\section{Antisite concentration dependence of Li diffusivity}

We follow the approach described in ref. ${ }^{10}$ to determine the Li diffusivity as a function of antisite concentration in $\mathrm{LiFePO}_{4}$ based on $\mathrm{Li}$ migration barriers calculated from the DFT calculations. ${ }^{7,10}$ In short, a 1D random walk model is set up to simulate the hopping of a $\mathrm{Li}$ ion between two $\mathrm{Fe}_{\mathrm{Li}}$ antisites within a [010] channel, which are separated by a distance $d$ that depends on the antisite concentration $p$ as $d=\left(1+p^{-1}\right) I_{\mathrm{b}} / 2$ where $I_{\mathrm{b}}$ is the lattice constant of LFP along [010]. The Li ion makes fast random jumps within the channel with an activation barrier of $270 \mathrm{meV}$ taken from ref. ${ }^{7}$ Whenever $\mathrm{Li}$ hops to a site next to an $\mathrm{Fe}_{\mathrm{Li}}$, it may cross over to the nearest migration channel to circumvent the obstruction of the antisite with an activation energy of $491 \mathrm{meV}$ as calculated by Malik et al. ${ }^{10}$ The frequencies of the two types of jumps are given by $\Gamma=v \exp \left(-E_{\mathrm{a}} / k T\right)$, where $v=10^{12} \mathrm{~s}^{-1}$ is the attempted frequency and $E_{\mathrm{a}}$ is the activation energy. With $\Gamma$ as input, 500 kinetic Monte Carlo simulations are run to estimate the average time $\langle t>$ it takes for the $\mathrm{Li}$ ion to escape the channel blocked by the two antisites. The Li diffusivity is calculated as $D=L^{2} /(2<$ $t>)$, in which $L=d / 2$ for $D_{[010]}, I_{a} / 2$ for $D_{[100]}$ and $I_{c} / 2$ for $D_{[001]}\left(I_{a} / I_{c}-\right.$ [100]/[001] lattice constants of LFP). We verified our calculation by reproducing the Li diffusivity values at $440 \mathrm{~K}$ and $D_{[010]}$ at $300 \mathrm{~K}$ reported in ref. ${ }^{10}$ and then used the method to determine $D_{[100]}, D_{[010]}$ and $D_{[001]}$ at different defect concentrations at $300 \mathrm{~K}$, which is shown in Supplementary Fig. 1.

\section{Phase-field model}

We use a previously reported phase-field model ${ }^{9,25}$ to simulate phase transformation in $\mathrm{LiFePO}_{4}$ upon Li intercalation, which is briefly described here. The site occupancy fraction of $\mathrm{Li}$ in $\mathrm{Li}_{c} \mathrm{FePO}_{4}, \mathrm{c}(r)$, serves as the field variable to distinguish between $\operatorname{LFP}(c=1)$ and $\operatorname{FP}(c=0)$ phases in the model. Li diffusion and the LFP $\leftrightarrow \mathrm{FP}$ phase transformation are described by the Cahn-Hilliard equation for $c(r)^{47,48}$

$\frac{\partial c}{\partial t}=-\nabla \cdot j=\nabla \cdot\left[\frac{\mathbf{D} V_{\mathrm{m}}}{R T} c(1-c) \nabla \mu_{\mathrm{Li}}\right]$

where the $\mathrm{Li}$ chemical potential $\mu_{\mathrm{Li}}$ is given by

$\mu_{\mathrm{Li}}=\frac{\partial f_{\text {chem }}(c)}{\partial c}-\kappa \nabla^{2} c=\mu_{\mathrm{Li}}^{\mathrm{eq}}+\frac{1}{V_{\mathrm{m}}}\left[R T \ln \frac{c}{1-c}+\Omega(1-2 c)\right]-\kappa \nabla^{2} c$

In Eqs. 6 and 7, D is the diffusion coefficient tensor, $V_{\mathrm{m}}=43.8 \mathrm{~cm}^{3} \mathrm{~mol}^{-1}$ is the molar volume of $\mathrm{LiFePO}_{4}{ }^{49} R$ is the gas constant and $\mu_{\mathrm{Li}}^{\text {eq }}$ is the equilibrium $\mathrm{Li}$ chemical potential at LFP/FP two-phase coexistence. A regular solution model is used to describe the homogeneous chemical free energy density $f_{\text {chem }}$ of $\mathrm{Li}_{\mathrm{c}} \mathrm{FePO}_{4}$ with $\Omega=12 \mathrm{~kJ} \mathrm{~mol}^{-1}{ }^{25}$ The gradient coefficient $\mathrm{K}$ is given a value of $1.68 \times 10^{-12} \mathrm{~J} \mathrm{~cm}^{-1}$, which produces a phase boundary energy of $0.072 \mathrm{~J} \mathrm{~m}^{-2}$ that averages the (100), (010) and (001) interface energies obtained from first-principles calculations. ${ }^{50}$

As the boundary condition, the Li intercalation flux at particle surface is described by the Butler-Volmer equation:

$j_{\mathrm{s}}=\frac{i_{0}}{F}\left[\exp \left(\frac{a V_{\mathrm{m}} \eta}{R T}\right)-\exp \left(-\frac{(1-a) V_{\mathrm{m}} \eta}{R T}\right)\right]$

where $\eta=\mu_{\mathrm{Li}}^{\mathrm{e}}-\mu_{\mathrm{Li}}$ is the surface reaction overpotential with $\mu_{\mathrm{Li}}^{\mathrm{el}}$ and $\mu_{\mathrm{Li}}$ being Li chemical potentials in the surrounding electrolyte and at particle surface, respectively. The underpotential $\Delta \phi$ is related to $\mu_{\mathrm{Li}}^{\mathrm{el}}$ as $\Delta \phi=F\left(\mu_{\mathrm{Li}}^{\mathrm{el}}-\mu_{\mathrm{Li}}^{\mathrm{eq}}\right) / V_{\mathrm{m}}$. We set $i_{0}=0.01 \mathrm{~A} \mathrm{~m}^{-2}$ (ref. ${ }^{32}$ ) and $a=0.5$ in Eq. 8. Supplementary Table 1 in SI summarizes the parameters used in the model and the sources of their values.
1D depth-averaged model

The model is derived in a similar way as in ref. ${ }^{13}$ Integrating Eq. 6 along the [010] axis, one has

$\frac{\partial \bar{c}}{\partial t}=\frac{1}{L_{[010]}} \int_{0}^{L_{0010]}} \frac{\partial}{\partial x}\left[\frac{D_{[100]} V_{\mathrm{m}}}{R T} c(1-c) \frac{\partial \mu_{\mathrm{Li}}}{\partial x}\right] d y+\frac{2 j_{\mathrm{s}}}{L_{[010]}}$

where $\bar{c}(x, t)=\int_{0}^{L_{[010]}} c(x, y, t) d y / L_{[010]}$ is the average Li concentration in the [010] direction. With the assumption of facile Li transport along [010] so that $c(r)$ is uniform along [010], i.e. $c(x, y, t)=\bar{c}(x, t)$, Eq. 7 becomes

$\mu_{\mathrm{Li}}(x, t)=\frac{\partial f_{\text {chem }}(\bar{c})}{\partial \bar{c}}-\kappa \frac{\partial^{2} \bar{c}}{\partial x^{2}}$

Accordingly, the 2D Cahn-Hilliard equation (Eq. 6) is reduced to the depthaveraged equation (Eq. 1), in which the overbar on $c$ is dropped.

Analytical solution to the depth-averaged model

An analytical expression of the traveling wave solution to the depthaveraged model can be derived with a few approximations. Applying the ansatz $c(x, t)=c\left(x-V_{\mathrm{PB}} t\right)$ to Eq. 1, one obtains

$-V_{\mathrm{PB}} \frac{d c}{d x}=\frac{\partial}{\partial x}\left[\frac{D_{[100]} V_{\mathrm{m}}}{R T} c(1-c) \frac{\partial \mu_{\mathrm{Li}}}{\partial x}\right]+\frac{2 j_{\mathrm{s}}}{L_{[010]}}$

The term on the left-hand side can be omitted from Eq. 11 when phase boundary migration is not very fast. In the sharp interface limit, the gradient term $\kappa d^{2} c / d x^{2}$ is removed from $\mu_{4 \mathrm{i}}$ in Eq. 10. This approximation is valid when the surface reaction zone width is much larger than the intrinsic thickness of the diffuse phase boundary. Equation 1 is thus simplified to

$D_{[100]} \frac{d^{2} c}{d x^{2}}+\frac{2 j_{s}}{L_{[010]}}=0$

Letting $x=0$ be the phase boundary location, Eq. 12 is completed by the following boundary conditions

$c\left(x=0_{-}\right)=c_{\mathrm{LFP}}^{\mathrm{eq}}, c\left(x=0_{+}\right)=c_{\mathrm{FP}}^{\mathrm{eq}}$

$c(x=-\infty)=c_{\mathrm{LFP}}(\Delta \phi), c(x=\infty)=c_{\mathrm{FP}}(\Delta \phi)$

where $c_{\mathrm{LFP}}^{\mathrm{eq}}$ and $c_{\mathrm{FP}}^{\mathrm{eq}}$ are LFP and FP compositions at two-phase equilibrium, and $c_{\mathrm{LFP}}(\Delta \phi)$ and $c_{\mathrm{FP}}(\Delta \phi)$ are the metastable LFP and FP compositions at underpotential $\Delta \phi$, respectively. By solving Eq. 12 through integration and applying Eq. 13, we obtain an implicit form of the traveling wave solution in the LFP $(x<0)$ and FP $(x>0)$ domains:

$K_{\mathrm{LFP}}(c) \equiv \int_{c_{\mathrm{LFP}}^{\mathrm{eq}}}^{c} \frac{d c^{\prime}}{\sqrt{l\left(c^{\prime}, \mathrm{L}_{\mathrm{LFP}}(\Delta \phi)\right)}}=-2 \sqrt{\frac{i_{0}}{F D_{[100 L} L_{0100}}} x, x<0$

$K_{\mathrm{FP}}(c) \equiv \int_{C_{\mathrm{FP}}^{\mathrm{eq}}}^{c} \frac{d c^{\prime}}{\sqrt{I\left(c^{\prime}, \mathrm{FPP}^{\prime}(\Delta \phi)\right)}}=2 \sqrt{\frac{i_{0}}{F D\left[1000 L_{0100]}\right.}} x, x>0$

Function $I$ is defined as $I\left(c_{1}, c_{2}\right) \equiv \int_{c_{1}}^{c_{2}} \hat{j}_{s}\left(c^{\prime}, \Delta \phi\right) d c^{\prime}$ and $\hat{j}_{s}=j_{s} /\left(i_{0} / F\right)$ is the dimensionless reaction flux. Inverting Eq. 14 , the solution is explicitly expressed as

$c(x)= \begin{cases}K_{\mathrm{LFP}}^{-1}\left(-2 \sqrt{\frac{i_{0}}{F D_{[100]} L_{[010]}}} x\right) & x<0 \\ K_{\mathrm{FP}}^{-1}\left(2 \sqrt{\frac{i_{0}}{F D_{[100]} L_{[010]}}} x\right) & x>0\end{cases}$

Supplementary Fig. 5 shows that the Li concentration profile predicted by Eq. 15 agrees very well the numerical solution.

As mentioned above, the surface reaction zone is defined as the region in which $\hat{j}_{s}(c)>a j_{s}^{\max }$, where $\hat{j}_{s}^{\max }=\hat{j}_{s}(c(x=0))$ is the peak reaction flux and $a$ is given a somewhat arbitrary value of 0.35 . Using Eq. 15, the surface reaction zone width $W$ is given by

$W=\left[K_{\mathrm{LFP}}\left(\hat{j}_{\mathrm{s}}^{-1}\left(a \hat{j}_{\mathrm{s}}^{\max }\right)\right)+K_{\mathrm{FP}}\left(\hat{j}_{\mathrm{s}}^{-1}\left(\alpha \hat{j}_{\mathrm{s}}^{\max }\right)\right)\right] \sqrt{\frac{F D_{[010]} L_{[010]}}{4 i_{0}}} \equiv \lambda_{1} \sqrt{\frac{D_{[010]} L_{[010]}}{i_{0}}}$

Calculating the phase boundary velocity $V_{\mathrm{PB}}$ from mass conservation, i.e. $V_{\mathrm{PB}}=2 \int_{-\infty}^{+\infty} j_{\mathrm{s}}(c(x)) d x /\left(\Delta c L_{[010]}\right)$, where $\Delta c=c_{\mathrm{LFP}}(\Delta \phi)-c_{\mathrm{FP}}(\Delta \phi)$, one obtains

$V_{\mathrm{PB}}=\frac{1}{\Delta c} \sqrt{\frac{i_{0} D_{[010]}}{F L_{[010]}}} \int_{-\infty}^{\infty}\left[\hat{j}_{s}\left(K_{\mathrm{LFP}}^{-1}(y)\right)+\hat{j}_{s}\left(K_{\mathrm{FP}}^{-1}(y)\right)\right] d y \equiv \lambda_{2} \sqrt{\frac{i_{0} D_{[010]}}{L_{[010]}}}$

Figure 4 shows that $W$ and $V_{P B}$ predicted by Eqs. 16 and 17 have very good agreement with the numerical solutions except at very small $D_{[100]}$, 
where $W$ is comparable to the diffuse phase boundary width and the sharp-interface assumption is not valid.

\section{DATA AVAILABILITY}

The data that support the findings of this study are available from the corresponding author upon request.

Received: 27 November 2018; Accepted: 12 November 2019; Published online: 06 December 2019

\section{REFERENCES}

1. Lee, J. et al. Unlocking the potential of cation-disordered oxides for rechargeable lithium batteries. Science 343, 519 (2014).

2. Kim, J., Lee, B., Kim, H., Kim, H. \& Kang, K. Redesign of $\mathrm{Li}_{2} \mathrm{MP}_{2} \mathrm{O}_{7}(\mathrm{M}=\mathrm{Fe}$ or $\mathrm{Mn})$ by tuning the Li diffusion in rechargeable battery electrodes. Chem. Mater. 28, 6894 (2016).

3. $\mathrm{Hu}$, J. et al. Tuning Li-ion diffusion in a-LiMn ${ }_{1-x} \mathrm{Fe}_{x} \mathrm{PO}_{4}$ nanocrystals by antisite defects and embedded $\beta$-phase for advanced Li-ion batteries. Nano Lett. 17, 4934 (2017).

4. Heenen, H. H., Scheurer, C. \& Reuter, K. Implications of occupational disorder on ion mobility in $\mathrm{Li}_{4} \mathrm{Ti}_{5} \mathrm{O}_{12}$ battery materials. Nano Lett. 17, 3884 (2017).

5. Xiong, $\mathrm{H}$. et al. Self-improving anode for lithium-ion batteries based on amorphous to cubic phase transition in $\mathrm{TiO}_{2}$ nanotubes. J. Phys. Chem. C. 116, 3181 (2012).

6. Xiong, $\mathrm{H}$. et al. Compositional tuning of structural stability of lithiated cubic titania via a vacancy-filling mechanism under high pressure. Phys. Rev. Lett. 110, 078304 (2013).

7. Morgan, D., Van der Ven, A. \& Ceder, G. Li conductivity in $\mathrm{Li}_{x} \mathrm{MPO}_{4}(\mathrm{M}=\mathrm{Mn}, \mathrm{Fe}$, Co, Ni) olivine materials. Electrochem. Solid-State Lett. 7, A30 (2004).

8. Amin, R., Maier, J., Balaya, P., Chen, D. P. \& Lin, C. T. Ionic and electronic transport in single crystalline $\mathrm{LiFePO}_{4}$ grown by optical floating zone technique. Solid State lon. 179, 1683 (2008).

9. Hong, L. et al. 2D Li diffusion behavior and probable hybrid phase transformation kinetics in olivine lithium iron phosphate. Nat. Commun. 8, 1194 (2017).

10. Malik, R., Burch, D., Bazant, M. \& Ceder, G. Particle size dependence of the ionic diffusivity. Nano Lett. 10, 4123 (2010).

11. Wong, L. L., Chen, H. M. \& Adams, S. Sodium-ion diffusion mechanisms in the low cost high voltage cathode material $\mathrm{Na}_{2+\delta} \mathrm{Fe}_{2-\delta / 2}\left(\mathrm{SO}_{4}\right)_{3}$. Phys. Chem. Chem. Phys. 17, 9186 (2015).

12. Kim, J. C., Seo, D. H., Chen, H. L. \& Ceder, G. The effect of antisite disorder and particle size on $\mathrm{Li}$ intercalation kinetics in monoclinic $\mathrm{LiMnBO}_{3}$. Adv. Energy Mater. 5, 1401916 (2015)

13. Singh, G. K., Ceder, G. \& Bazant, M. Z. Intercalation dynamics in rechargeable battery materials: general theory and phase-transformation waves in $\mathrm{LiFePO}_{4}$. Electrochim. Acta 53, 7599 (2008).

14. Chen, L.-Q. Phase-field models for microstructure evolution. Annu. Rev. Mater. Res. 32, 113 (2002).

15. Boettinger, W. J., Warren, J. A., Beckermann, C. \& Karma, A. Phase-field simulation of solidification. Annu. Rev. Mater. Res. 32, 163 (2002).

16. Moelans, N., Blanpain, B. \& Wollants, P. An introduction to phase-field modeling of microstructure evolution. Calphad 32, 268-294 (2008).

17. Tang, M. et al. Model for the particle size, overpotential, and strain dependence of phase transition pathways in storage electrodes: application to nanoscale olivines. Chem. Mater. 21, 1557 (2009).

18. Cogswell, D. A. \& Bazant, M. Z. Coherency strain and the kinetics of phase separation in $\mathrm{LiFePO}_{4}$ nanoparticles. ACS Nano 6, 2215 (2012).

19. Heo, T. W., Chen, L. Q. \& Wood, B. C. Phase-field modeling of diffusional phase behaviors of solid surfaces: a case study of phase-separating $\mathrm{Li}_{x} \mathrm{FePO}_{4}$ electrode particles. Comput. Mater. Sci. 108, 323 (2015).

20. Zhang, W. et al. Localized concentration reversal of lithium during intercalation into nanoparticles. Sci. Adv. 4, eaao2608 (2018).

21. Heo, T. W., Tang, M., Chen, L. Q. \& Wood, B. C. Defects, entropy, and the stabilization of alternative phase boundary orientations in battery electrode particles. Adv. Energy Mater. 6, 1501759 (2016).

22. Chen, G., Song, X. \& Richardson, T. J. Electron microscopy study of the $\mathrm{LiFePO}_{4}$ to $\mathrm{FePO}_{4}$ phase transition. Electrochem. Solid-State Lett. 9, A295 (2006).

23. $\mathrm{Li}, \mathrm{Y}$. et al. Current-induced transition from particle-by-particle to concurrent intercalation in phase-separating battery electrodes. Nat. Mater. 13, 1149 (2014).

24. Laffont, L. et al. Study of the $\mathrm{LiFePO}_{4} / \mathrm{FePO}_{4}$ two-phase system by high-resolution electron energy loss spectroscopy. Chem. Mater. 18, 5520 (2006).

25. Tang, M., Belak, J. F. \& Dorr, M. R. Anisotropic phase boundary morphology in nanoscale olivine electrode particles. J. Phys. Chem. C. 115, 4922 (2011).
26. Liu, H. et al. Capturing metastable structures during high-rate cycling of $\mathrm{LiFePO}_{4}$ nanoparticle electrodes. Science 344, 1252817 (2014).

27. Delmas, C., Maccario, M., Croguennec, L., Le Cras, F. \& Weill, F. Lithium deintercalation in $\mathrm{LiFePO}_{4}$ nanoparticles via a domino-cascade model. Nat. Mater. 7, 665 (2008).

28. Christensen, J. \& Newman, J. Stress generation and fracture in lithium insertion materials. J. Solid State Electrochem. 10, 293 (2006).

29. Woodford, W. H., Chiang, Y.-M. \& Carter, W. C. "Electrochemical shock" of intercalation electrodes: a fracture mechanics analysis. J. Electrochem. Soc. 157, A1052 (2010).

30. Woodford, W. H., Carter, W. C. \& Chiang, Y.-M. Design criteria for electrochemical shock resistant battery electrodes. Energy Environ. Sci. 5, 8014 (2012).

31. Wang, J., Karen Chen-Wiegart, Y. C., Eng, C., Shen, Q. \& Wang, J. Visualization of anisotropic-isotropic phase transformation dynamics in battery electrode particles. Nat. Commun. 7, 12372 (2016).

32. Lim, J. et al. Origin and hysteresis of lithium compositional spatiodynamics within battery primary particles. Science 353, 566 (2016).

33. $\mathrm{Li}$, Z. et al. [100]-Oriented $\mathrm{LiFePO}_{4}$ nanoflakes toward high rate li-ion battery cathode. Nano Lett. 16, 795 (2016).

34. Li, Z. et al. Orientation-dependent lithium miscibility gap in $\mathrm{LiFePO}_{4}$. Chem. Mater. 30, 874 (2018).

35. Zhang, $X$. et al. Rate-induced solubility and suppression of the first-order phase transition in olivine $\mathrm{LiFePO}_{4}$. Nano Lett. 14, 2279 (2014).

36. Hess, M., Sasaki, T., Villevieille, C. \& Novak, P. Combined operando X-ray diffraction-electrochemical impedance spectroscopy detecting solid solution reactions of $\mathrm{LiFePO}_{4}$ in batteries. Nat. Commun. 6, 8169 (2015).

37. Malik, R., Zhou, F. \& Ceder, G. Kinetics of non-equilibrium lithium incorporation in $\mathrm{LiFePO}_{4}$. Nat. Mater. 10, 587 (2011).

38. Bai, P., Cogswell, D. A. \& Bazant, M. Z. Suppression of phase separation in $\mathrm{LiFePO}_{4}$ nanoparticles during battery discharge. Nano Lett. 11, 4890 (2011).

39. Bae, C. J., Erdonmez, C. K., Halloran, J. W. \& Chiang, Y. M. Design of battery electrodes with dual-scale porosity to minimize tortuosity and maximize performance. Adv. Mater. 25, 1254 (2013).

40. Sander, J. S., Erb, R. M., Li, L., Gurijala, A. \& Chiang, Y. M. High-performance battery electrodes via magnetic templating. Nat. Energy 1, 16099 (2016).

41. Gallagher, K. G. et al. Optimizing areal capacities through understanding the limitations of lithium-ion electrodes. J. Electrochem. Soc. 163, A138 (2016).

42. Li, Y. Y. et al. Fluid-enhanced surface diffusion controls intraparticle phase transformations. Nat. Mater. 17, 915 (2018).

43. Dargaville, S. \& Farrell, T. W. The persistence of phase-separation in $\mathrm{LiFePO}_{4}$ with two-dimensional $\mathrm{Li}^{+}$transport: the Cahn-Hilliard-reaction equation and the role of defects. Electrochim. Acta 108, 876 (2013).

44. Chen, J. J. \& Graetz, J. Study of antisite defects in hydrothermally prepared $\mathrm{LiFePO}_{4}$ by in situ X-ray diffraction. ACS Appl. Mater. Inter. 3, 1380 (2011).

45. Zou, Y. H. et al. Suppressing Fe-Li Antisite defects in $\mathrm{LiFePO}_{4} /$ carbon hybrid microtube to enhance the lithium ion storage. Adv. Energy Mater. 6, 1601549 (2016).

46. Chung, S.-Y., Choi, S.-Y., Yamamoto, T. \& Ikuhara, Y. Atomic-scale visualization of antisite defects in LiFePO . Phys. Rev. Lett. 100, 125502 (2008).

47. Cahn, J. W. \& Hilliard, J. E. Free energy of a nonuniform system .1 Interfacial free energy. J. Chem. Phys. 28, 258 (1958).

48. Cahn, J. W. On spinodal decomposition. Acta Metall. 9, 795 (1961)

49. Meethong, N., Huang, H. Y. S., Speakman, S. A., Carter, W. C. \& Chiang, Y. M. Strain accommodation during phase transformations in olivine-based cathodes as a materials selection criterion for high-power rechargeable batteries. Adv. Funct. Mater. 17, 1115 (2007).

50. Abdellahi, A., Akyildiz, O., Malik, R., Thornton, K. \& Ceder, G. Particle-size and morphology dependence of the preferred interface orientation in $\mathrm{LiFePO}_{4}$ nanoparticles. J. Mater. Chem. A. 2, 15437 (2014).

\section{ACKNOWLEDGEMENTS}

K.Y. and M.T. are supported by DOE under project number DE-SC0019111. L.H. was supported by DOE under project number DE-SC0014435. We acknowledge supercomputing allocations provided by the Texas Advanced Computing Center (TACC) at The University of Texas, and the National Energy Research Scientific Computing Center, a DOE Office of Science User Facility supported by the Office of Science of the U.S. Department of Energy under Contract No. DE-AC02-05CH11231.

\section{AUTHOR CONTRIBUTIONS}

M.T. conceived the project. K.Y. and L.H. performed the phase-field simulations. K.Y. and M.T. carried out the theoretical analysis and the kinetic Monte Carlo simulations. M.T., L.H. and K.Y. wrote the paper. 


\section{COMPETING INTERESTS}

The authors declare no competing interests.

\section{ADDITIONAL INFORMATION}

Supplementary information is available for this paper at https://doi.org/10.1038/ s41524-019-0255-3.

Correspondence and requests for materials should be addressed to M.T.

Reprints and permission information is available at http://www.nature.com/ reprints

Publisher's note Springer Nature remains neutral with regard to jurisdictional claims in published maps and institutional affiliations.
Open Access This article is licensed under a Creative Commons Attribution 4.0 International License, which permits use, sharing, adaptation, distribution and reproduction in any medium or format, as long as you give appropriate credit to the original author(s) and the source, provide a link to the Creative Commons license, and indicate if changes were made. The images or other third party material in this article are included in the article's Creative Commons license, unless indicated otherwise in a credit line to the material. If material is not included in the article's Creative Commons license and your intended use is not permitted by statutory regulation or exceeds the permitted use, you will need to obtain permission directly from the copyright holder. To view a copy of this license, visit http://creativecommons. org/licenses/by/4.0/.

(c) The Author(s) 2019 